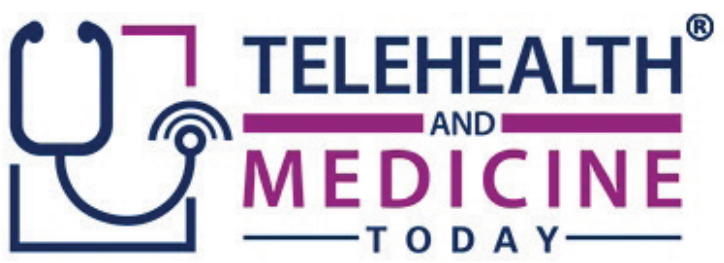

\title{
Maximizing VA remote patient monitoring during the COVID-19 response
}

Catherine Buck, MSN, GNP-BC ${ }^{1}$; Rita F. Kobb, MSN, GNP-BC ${ }^{2}$; Ron Sandreth, RD1; Lisa Alexander, RN-BC, MSN³; Sherron Olliff, MSW, LCSW 3 ; Carol Westfall, RN, MSN ${ }^{4}$; Carla L. Anderson MSN, RN $;$; A. Laurie Graaff, RN, MBA, MS-ISM ${ }^{6}$; Joseph Giovannucci ${ }^{7}$; Aszur Rollins ${ }^{8}$

Affiliations: ${ }^{1}$ Remote Patient Monitoring - Home Telehealth, US Department of Veterans Affairs, Office of Connected Care; ${ }^{2}$ VA Office of Connected Care Quality and Training Division, US Department of Veterans Affairs, Office of Connected Care, Quality and Training Division; ${ }^{3}$ Remote Patient Monitoring - Home Telehealth, US Department of Veterans Affairs, Office of Connected Care, Quality and Training Division; ${ }^{4}$ Disease Management Protocol (DMP) Oversight, US Department of Veterans Affairs, Office of Connected Care, Quality and Training Division; ${ }^{5}$ US Department of Veterans Affairs, Office of Connected Care, Quality and Training Division; ${ }^{6}$ Telehealth Emergency Management Program, US Department of Veterans Affairs, Office of Connected Care, Quality and Training Division; ${ }^{7}$ Telehealth Program, Denver Logistics Center (DLC), US Department of Veterans Affairs, Office of Acquisition and Logistics; ${ }^{8}$ US Department of Veterans Affairs, Office of Healthcare Transformation

Corresponding Author: Catherine Buck, Email: catherine.buck@va.gov

Keywords: COVID-19 pandemic; remote patient monitoring; home telehealth; VA; Veteran's administration.

Section: Training Tools for Health Professionals \& Patients

Objective: The Veterans Health Administration (VHA) has one of the largest remote patient monitoring programs in the United States and is supported by an enterprise-wide infrastructure for providers, clinicians, staff, Veterans, and caregivers. The coronavirus disease 2019 (COVID-19) pandemic, however, presented new challenges: a sudden need to provide large-scale remote monitoring for a new disease that did not yet have a disease management protocol (DMP). Veterans Health Administration (VHA) needed to be ready within weeks to provide this daily monitoring for hundreds - even thousands - of Veterans.
Methods: The US Department of Veterans Affairs Office of Connected Care already had a comprehensive infrastructure in place for its remote patient monitoring - Home Telehealth (RPM-HT) program. Connected Care activated and built on this infrastructure to support providers, clinicians, and staff in their efforts to rapidly bring RPM - HT to Veterans across the nation when they had COVID-19 symptoms or exposure. To do this, Connected Care activated an emergency management plan, rapidly developed a new COVID-19-specific DMP, added weekend monitoring, and procured 
critically needed monitoring supplies, such as thermometers and pulse oximeters. The strong foundation for Connected Care allowed for innovation and flexibility, such as the training of non-RPM-HT staff in RPM-HT processes, $R P M-H T$ enrollment within acute care settings, and new strategic partnerships.

Outcomes: More than 23,500 Veterans were enrolled for COVID-19-related monitoring from March 2020 to May 2021. During December 2020 and January 2021, the number of Veterans being monitored in a single day topped 2,000. Even with this rapid buildup, patient satisfaction levels remained at about $90 \%$ in numerous categories. In addition, the percentage of Veterans admitted to Veterans Administration (VA) facilities while on COVID-19-related home monitoring has been extremely low, at 4\%, the monitoring system has been a potential indicator in enabling Veterans who did have the virus to convalesce at home. Further study is needed to determine the impact RPM - HT enrollment for COVID-19 care had on the need for inpatient care.

Conclusion: The Office of Connected Care's established, enterprise-wide RPM-HT business, clinical, and technical infrastructure enabled VHA to enter the COVID-19 public health emergency well-positioned for the rapid deployment and growth of at-home and mobile monitoring. As the COVID-19 emergency made at-home management of Veterans increasingly important, the national RPM - HT program successfully adapted its practices to meet the needs of Veterans, caregivers, and staff.

$\mathrm{T}$ The Veterans Health Administration (VHA) - a component of the US Department of Veterans Affairs - is the largest integrated healthcare system in the United States, which serves more than 8.5 million Veterans annually, including many aged 65 and older. ${ }^{1}$ From the beginning of the coronavirus disease 2019 (COVID-19) pandemic through April 2021, more than 250,000 Veterans, non-Veteran patients, and employees were tested or treated at a VA facility for known or probable COVID-19. ${ }^{2}$ Veterans are particularly vulnerable because they are more likely to be chronically ill, with multiple comorbidities, than their private-sector counterparts. Veterans would be at greater risk of serious outcomes if infected by the virus.

The Remote Patient Monitoring - Home Telehealth (RPM - HT) program, under Telehealth services in the Office of Connected Care, has played a critical role in helping VHA meet the demand for COVID-19 care, provide the best standard of care to Veterans, and relieve any unnecessary strain on VHA facilities.

VHA has experienced increased growth in its RPM - HT programs during the pandemic, particularly in using disease management protocols (DMPs) specifically developed to monitor Veterans with COVID-19 symptoms or exposure. These protocols were used with existing remote monitoring in-home and mobile technologies. From March 2020 through May 10, 2021, more than 23,500 Veterans were enrolled in RPM - HT programs for COVID-19-related monitoring, with estimated 246,000 daily sessions being completed. These sessions equate to clinical touch points that might otherwise have had to occur in person, so the use of RPM - HT has helped reduce the burden on other VA resources, such as primary care clinics, emergency departments, and inpatient facilities. ${ }^{3}$

There was a critical need for RPM - HT programs especially in the early stages of the pandemic and during subsequent surges in infections. These surges resulted in a daily RPM - HT census peak of just over 2,000 in mid-December 2020 and mid-January 2021 (Fig. 1). The need closely 


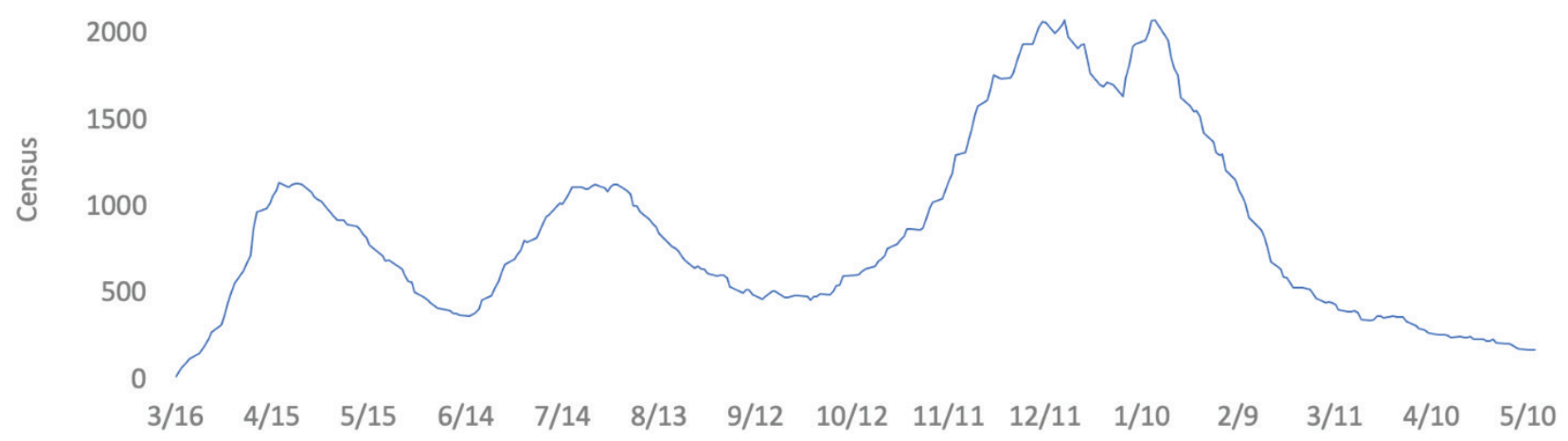

Figure 1-Daily census of RPM - HT enrollment for COVID-19-associated disease management protocols, March 16, 2020 to May 10, 2021.

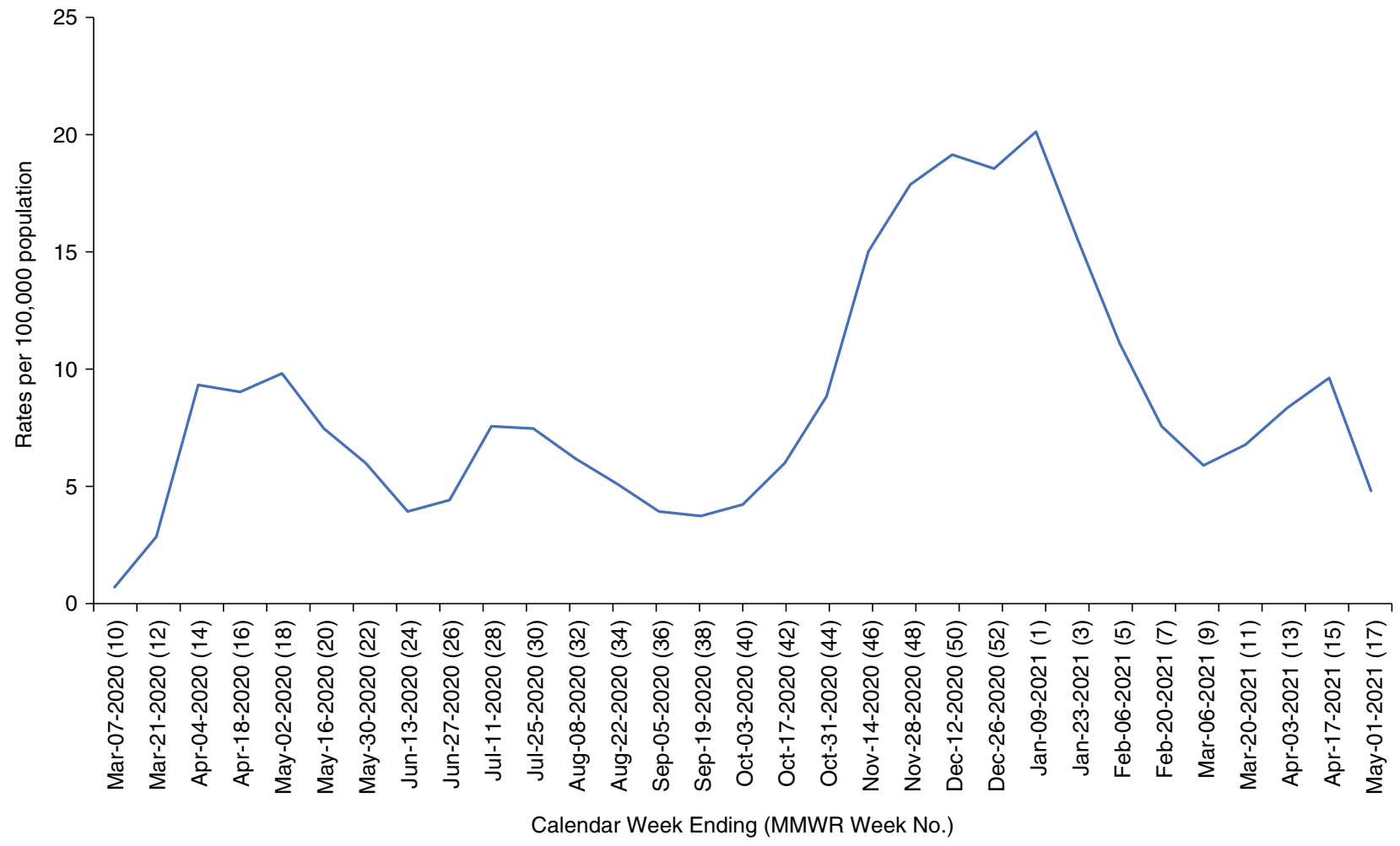

Figure 2-US population rate of COVID-19-associated hospitalizations.

Source: Morbidity and Mortality Weekly Report, Centers for Disease Control and Prevention.

tracked with what was occurring nationwide in terms of COVID-associated hospitalizations (Fig. 2) and was widely distributed over VHA's regional healthcare networks, known as Veterans Integrated Service Networks (VISNs) (Figs. 3 and 4$){ }^{3}$
The ability of VHA to ramp up its use of RPM HT program to meet this need was enabled by the existing national communications, training, resource management, and equipment distribution infrastructure it had developed since 2003 for clinical staff, Veterans, and caregivers. 


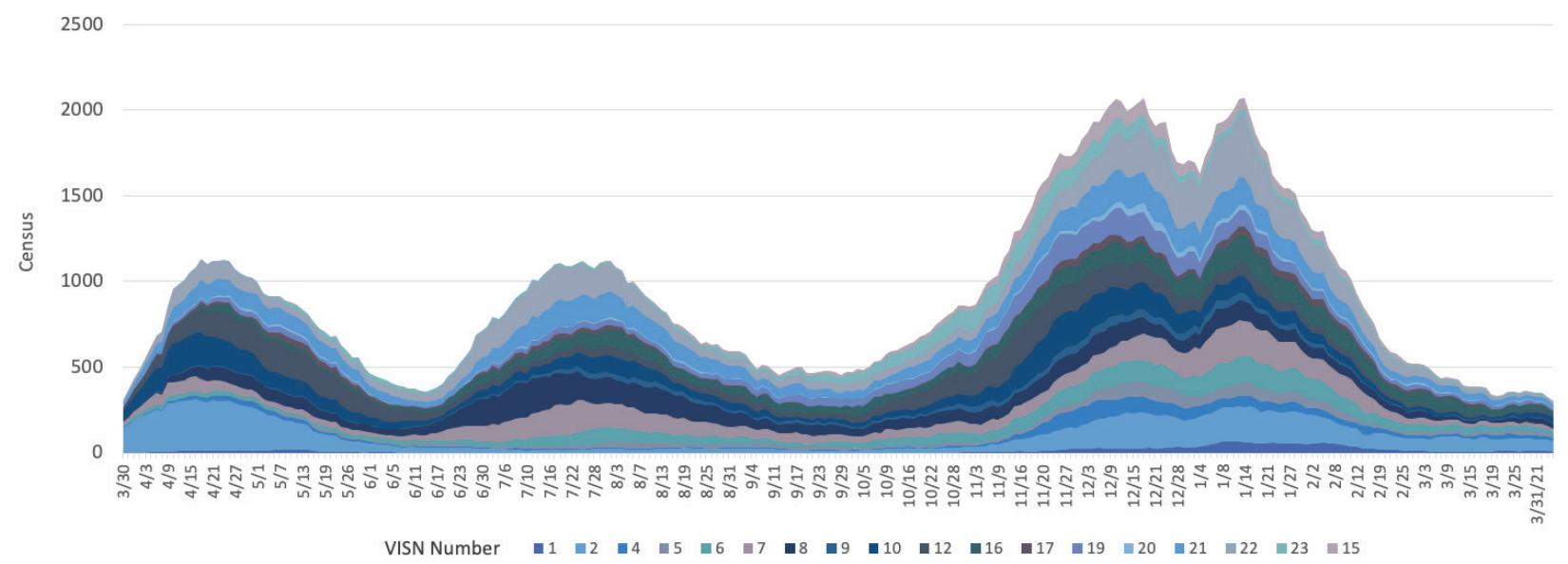

Figure 3-Daily census of RPM - HT enrollment for COVID-19-associated disease management protocols by Veterans Integrated Service Network, March 30, 2020, to March 31, 2021.

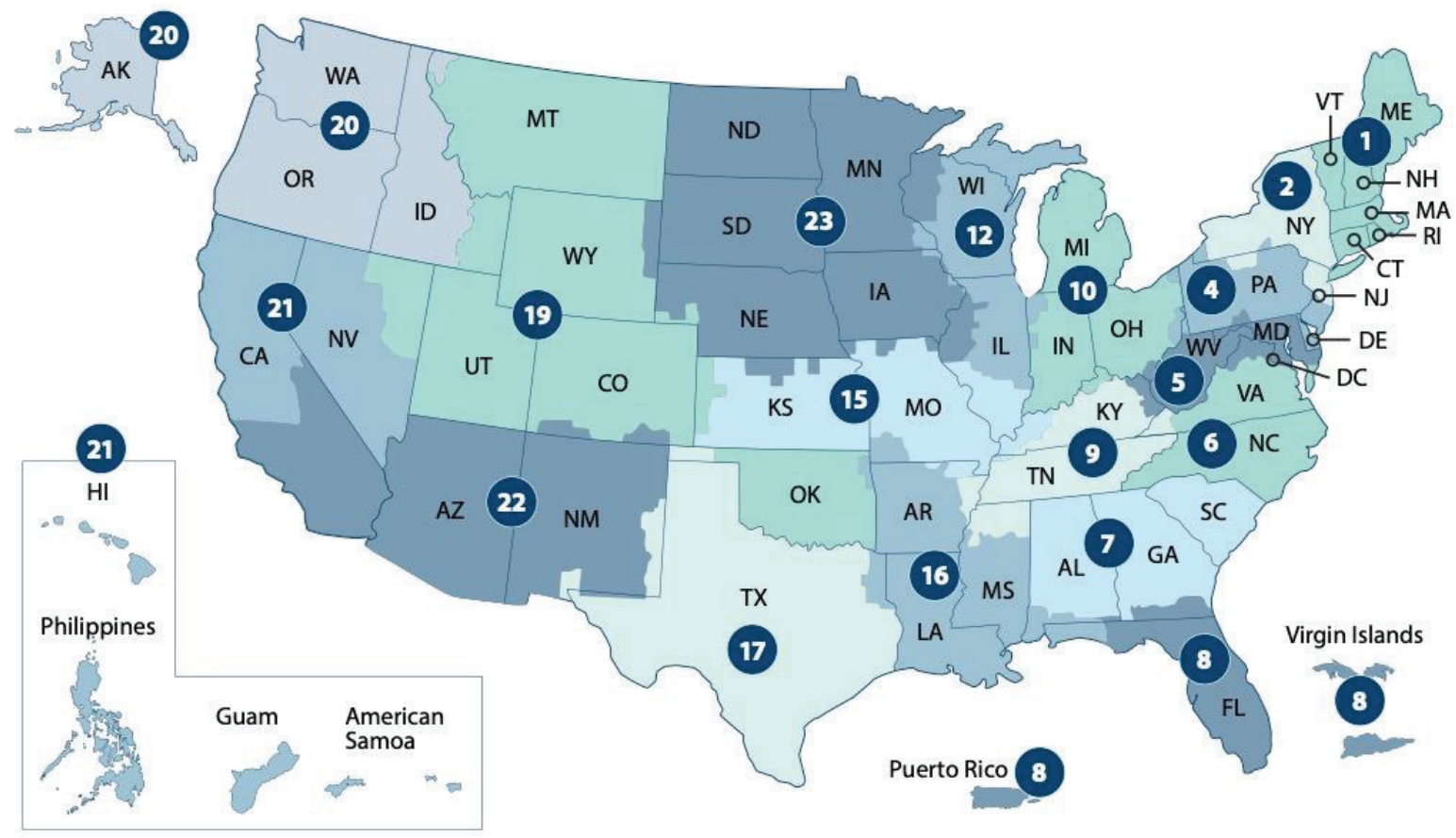

Figure 4-Map showing the geographic area of each Veterans Integrated Service Network.

These RPM - HT processes were in place before the COVID-19 pandemic, and rapidly expanded and adjusted to meet the needs of clinicians and Veterans during the pandemic. This research article describes VHA's RPM - HT infrastructure and how it leveraged an established enterprisewide program to optimize the agency's response to the COVID-19 pandemic. $^{4-6}$ 


\section{RPM - HT: BACKGROUND AND OVERVIEW}

Since its inception in July 2003, VHA's RPM HT program has progressively expanded in number of Veterans served and innovative technologies used. At the same time, the mission of the program has remained consistent: disease prevention, health promotion, symptom management, and Veteran self-management through education. RPM - HT applies care and case management principles to coordinate care using health informatics, disease management, and technologies, such as in-home and mobile monitoring, messaging, and video technologies. These strategies are used to provide the right care, in the right place, at the right time. ${ }^{7-9}$

RPM - HT is now a routine service supporting care for Veterans with both acute and chronic conditions. Since its beginning, the program has used evidence-based DMPs, such as the ones used for COVID-19 patients. There are more than 20 VHA standardized DMPs - including ones for hypertension, diabetes, heart failure, chronic obstructive pulmonary disease, and depression - in addition to RPM - HT vendorprovided DMPs that cover a wide range of conditions, as well as health promotion. These DMPs cover disease education, self-care management, reporting of symptoms and vital sign data, among other topics necessary for monitoring a particular condition. ${ }^{7-9}$

Veterans are enrolled in the RPM - HT program in coordination with their provider and are screened for appropriateness using specific enrollment inclusion and exclusion criteria. After a Veteran is identified as an appropriate candidate for the program, a full initial assessment and treatment plan is completed by a Care Coordinator in collaboration with the Veteran's care team. Care Coordinators are licensed healthcare professionals - primarily registered nurses, nurse practitioners, social workers, and registered dietitians. In 2003, the program had 110 Care Coordinators managing 1,500 Veterans. There are currently more than 2,800 Care Coordinators managing more than 75,000 Veterans. $^{7-9}$

Based on the initial assessment and specific Veteran need, Care Coordinators determine the best technology to assign and provide to the Veterans. Two contracted vendors provide these technologies, which include a video hub device (a physical unit with a screen), interactive voice response (IVR) (using phone voice and keypad responses), a web browser, a tablet, and apps. In addition, a wide range of peripheral devices collect and transmit data by wired, Bluetooth, or manual entry. The technologies are ordered, distributed, and refurbished through a centralized process.

When a Veteran is enrolled in the RPM - HT program, daily DMP sessions capture biometric data and symptom responses, transmit data via secure information technology processes, and provide health education. Rather than capturing biometric data alone, the unique DMPs provide a comprehensive review of the Veteran's health status. Although these innovative technologies are important, providing the information needed to monitor large panels of Veterans, the heart of the program is the personal connection of the Veterans with their assigned Care Coordinator and the intensive case management provided. This results in reduced hospital admissions and bed days of care, improved clinical outcomes, and a high level of patient satisfaction for home telehealth patients. ${ }^{7}$

\section{COVID-19 RPM - HT MOBILIZATION} In February 2020, as the COVID-19 outbreak worsened, VHA's Office of Connected Care rapidly began leveraging the framework of the 
RPM - HT program to prepare and care for Veterans. The director and deputy director of the national RPM - HT program, in collaboration with Connected Care leadership, immediately identified several critical areas of need and coordinated strategic and collaborative efforts. These critical areas, discussed separately below, included communications, leveraging of existing technologies, activation of emergency management plans, COVID-19 DMP development, 7-day-a-week staffing coverage, and logistical technology management and procurement.

\section{Communications}

Detailed, frequent, and two-way communications for all RPM - HT staff and programs across VA were critical to the successful implementation of COVID-19-related monitoring strategies. These communications included a white paper, weekly email updates, a weekly open forum, and a new intranet page.

\section{White Paper}

The first communication was a March 4, 2020, white paper, 'Use of Remote Patient Monitoring - Home Telehealth Technologies for COVID-19', which summarized current and planned strategies at the start of the pandemic. This communication, sent by RPM - HT leadership to all RPM - HT staff and network Telehealth Program Managers via the well-established national alert email communication process, was one of the first national COVID-19 guidance documents developed and sent by any program staff in VA. The white paper provided the following:

- gave a brief overview of the RPM - HT program, which was especially critical for non-RPM - HT staff.

- described appropriate use of DMP for COVID-19 monitoring and plans for COVID19-specific DMP development.
- detailed the activation of emergency plans.

- recommended the use of IVR for RPM - HT sessions.

- guided readers to follow the usual documentation processes, incorporating any facility-mandated COVID-19-specific templates, but added a key distinction: when enrolled, each Veteran is routinely assigned a Category of Care that identifies Veteran's level of care or acuity. For COVID-19 monitoring, staff were instructed to use the rarely utilized Acute Care Management category, which helped distinguish COVID19 patients for tracking and data purposes.

\section{Weekly Updates}

The white paper was just the first of many communications that Telehealth services provided throughout the pandemic. Well-established national communication processes in the RPM - HT program became a critical factor for rapidly providing information at the onset of the COVID-19 pandemic.

An RPM - HT national email alert process, which provides communication to all VA RPM - HT staff across the country to inform them of technology issues, clinical updates, or program process changes, has been used for many years with great success and customer satisfaction. From the start of the COVID-19 crisis, this process was leveraged to provide frequent and at least weekly updates throughout the pandemic. These regular updates were critical because of the evolving guidance not only from VA but also from the Centers for Disease Control and Prevention (CDC) regarding the care of COVID-19 patients. The weekly updates also provided enrollment data, documentation, and coding guidance, as well as information on any logistical concerns about the availability of technology, such as thermometers and pulse oximeters. 


\section{Weekly Open Forum}

One of the most successful communication methods employed was a weekly COVID-19 open forum for all staff. This provided an opportunity for the national office to review weekly updates and, more importantly, provided a forum in which staff could discuss leading practice, raise concerns, and ask questions. Often, these weekly forums helped give the national program lead insights on additional training needs or on process gaps to be addressed.

\section{Intranet Page}

Telehealth services also created an RPM - HT COVID-19 page on the VHA Telehealth Services intranet site to provide just-in-time information and serve as a staff reference point for COVID-19 information.

\section{LEVERAGING EXISTING TECHNOLOGIES}

One of the critical items included in the white paper was the recommendation to use IVR, which delivers sessions to Veterans through their own landline or mobile phones. With IVR, responses are entered via voice or keypad and are then transmitted securely. Before the pandemic, IVR was used for about $25 \%$ of Veterans enrolled in RPM - HT programs.

Having IVR as a long-established contracted technology was important for the rapid scale-up of RPM - HT to address the needs of monitoring COVID-19. Because IVR does not involve a hub device, which would have to be provided and sent to Veterans, Veterans using IVR could begin to participate in the program more quickly. In addition, the use of IVR reduced the burden on the centralized distribution staff at the Denver Logistics Center (which centrally manages RPM - HT supplies), especially early in the rapid scale-up for COVID-19 monitoring. Although infection control procedures are routinely followed for all refurbished hub devices, the use of IVR reassured any staff or Veterans concerned about the reuse of devices; this reassurance was particularly important for a program for Veterans who were being monitored for COVID-19.

Given the expectation of large numbers of enrollments, the use of IVR also represented using VA resources wisely during the pandemic, as it was substantially less costly than home hub devices.

\section{EMERGENCY MANAGEMENT PLAN ACTIVATION}

All RPM - HT programs are required to develop and regularly test emergency plans for natural or health disasters, such as the COVID-19 pandemic. These plans identify the following points:

- Staffing coverage needs, either within or outside of VA networks.

- Global messaging to be sent to enrolled Veterans via their technology.

- Risk stratification of Veterans into three categories - high, moderate, and low - based on their healthcare needs and complexity of care, which is used to determine the need for phone calls directly to Veterans. The risk system is based on the number of days of emergency and how the loss of power, water, and access to care might affect the Veteran's health condition.

The following are the specific examples of how the well-established emergency management plans were used during the COVID-19 pandemic:

- Reviewing panels of currently enrolled Veterans to determine whether any were appropriate for discharge, to free space in facilities for the potentially large number of Veterans who might need to be admitted for COVID-19-related care. 
- Identifying 'sister sites' to assist with staff coverage if needed. This was particularly helpful for sites in outbreak areas and resulted in coverage within VISNs and even across the networks.

- Encouraging teleworking as a tool to reduce potential exposure of staff to the coronavirus and to maximize the number of staff available to care for Veterans.

- Employing the established global messaging feature of RPM - HT technologies to rapidly provide critical COVID-19 information on infection control, symptoms, and when to seek care to all RPM - HT-enrolled Veterans.

\section{COVID-19 DMP DEVELOPMENT}

In February 2020, as concerns over the spread of the virus increased, one of the critical identified gaps was the lack of a DMP specific to COVID-19 care.

Until that gap could be closed, guidance recommended using currently available DMPs, such as those for hypertension and chronic obstructive pulmonary disease, for COVID-19 monitoring. With all DMPs, Care Coordinators can assign specific biometric data collection, so temperature and pulse oximetry could be added to these DMPs if they were not already included.

At the same time, work began on a new DMP. Because of the resources required for developing, and maintaining updates to, DMPs, it was decided to make the content appropriate for any infectious illness instead of that specific to COVID-19. That way, the DMP could also be used for other widespread healthcare events, such as influenza outbreaks or other pandemics. The content of the DMP would be general enough to use for any infectious illness; however, it would also guide users to refer to the CDC website for updates specific to COVID-19.
As previously noted, there are two main types of DMPs used in the RPM - HT program: vendor developed and VHA standardized. VHA DMPs typically take a longer time to develop and have a more involved implementation process, including review and approvals by VA subject matter experts, as well as vendor contractual implications. Telehealth services leadership understood early on that vendordeveloped DMPs would be the quickest path to having a DMP available for COVID-19 monitoring. Therefore, a Telehealth services contract representative approached the two RPM - HT vendors regarding the need. In a highly commendable fashion, the first of these vendor DMPs was ready on March 5, 2020 6 days before the pandemic was formally declared.

In parallel, VHA began work on a VHA standardized Infectious Illness DMP. To begin this process, the team in charge of overseeing current DMPs immediately set up daily calls. Usually, it can take anywhere from 9 to 12 months to years to launch a fully developed DMP, and VHA had not been developing DMPs for several years when called on to do this one; however, VHA was able to make the new Infectious Illness DMP available within weeks on April 7, 2020.

Several factors contributed to this DMP's rapid completion.

Typically, the development of DMP is a lengthy process because of the collaboration with identified subject matter experts and the need for consensus on the final content. This final content usually comes from existing national clinical guidelines. For the infectious illness DMP, the CDC website was the single source of truth for content, making this part of the process much easier and faster. 
Other factors that expedited completion included the following:

- use of a very experienced DMP development team

- collaboration with Telehealth leadership

- expedited availability of resources

- vendor participation.

Subject matter experts involved in this DMP's development included members of VA's COVID19 Emergency Management Coordination Cell and the Offices of Emergency Management and Infectious Disease. VHA and vendor DMPs were regularly updated to align with changing $\mathrm{CDC}$ guidance. The VHA Infectious Illness DMP will be reviewed annually, which is the standard for all VHA standardized DMPs.

\section{SEVEN-DAY-A-WEEK MONITORING AND STAFFING}

RPM - HT program standards include monitoring during normal business hours (8 a.m.-4:30 p.m.), Monday to Friday. Veterans are instructed upon enrollment to contact urgent care or call 911, as appropriate, particularly outside the normal business hours of the program. During the development of the DMPs for COVID-19 use, however, the subject matter experts recommended that the monitoring activities of the RPM - HT program be extended to 7 days a week, including 8 a.m. to 4:30 p.m. monitoring on weekends. This was due to the potential for rapid changes and decline in Veterans with COVID-19.

An April 24, 2020, national memo, titled 'COVID-19: Increased Remote Patient Monitoring -Home Telehealth Staffing Needs to Expand Care to COVID-19 Patients', was developed by Telehealth services and issued through the VA Office of the Assistant Under Secretary for Health for Clinical Services to all VISN directors. This memo directed all
RPM - HT programs to have plans in place by May 1 to add weekend hours. To help accommodate this change, VHA decided to train and make use of non-RPM - HT staff, such as those working in outpatient clinics that were closed at the time because of the pandemic. To accomplish this, the Telehealth services training center developed a new training for non-RPM - HT staff, which allowed for an abbreviated orientation and timely onboarding.

\section{TECHNOLOGY MANAGEMENT AND PROCUREMENT}

An additional challenge early in the pandemic was the procurement of needed technologies, particularly thermometers and pulse oximeters (for measurement of blood oxygen levels), that were critical for the assessment of the clinical status of monitored COVID-19 patients. The following steps were taken to help address this issue:

- The Telehealth Services deputy lead had daily discussions and formal weekly meetings with the customer support team at the Denver Logistics Center to review supply chain issues to ensure that there was available stock of needed devices.

- A tracking tool was developed with increased minimum stock levels and supply expectancy for higher use devices, such as thermometers and pulse oximeters.

- The Denver Logistics Center worked with vendors to ensure a stock level of at least 1,000 thermometers and pulse oximeters each, at any given moment.

This forecasting and collaboration enabled the Denver Logistics Center to acquire an inventory in advance of peak times and to efficiently prepare for supply chain shortages. In addition, the center worked with infectious disease control experts and manufacturers to ensure that ongoing 
practices for controlling pre-COVID-19 infection were still appropriate.

Later in the pandemic, starting in fall 2020, the logistics center worked to refurbish thermometers and pulse oximeters and to offer them at a discounted rate to ensure increased stock levels in a cost-effective manner. This led to an abundance of stock going into spring 2021, preparing the program for future outbreaks.

\section{TRAINING}

The Office of Connected Care had a successful, competency-based training program in place long before the pandemic through its Quality and Training (QT) division. Currently, the main platform for training is the Connected Care Academy, which operates on the Blackboard learning management system. Through the academy, the QT division offers self-paced courses, as well as live events, to provide greater accessibility and distribution of training content. All VHA staff can have access to the academy, and non-VA staff can access it through a self-registration process.

The QT division has ample experience in developing new trainings quickly, which is often necessary to keep up with changes in technology, DMPs, or VHA priorities.

The QT division's existing training infrastructure enabled VHA to respond quickly with new education and training for providers, clinicians, and staff involved in enrolling and monitoring Veterans with COVID-19 or COVID-19-like symptoms. The new educational strategies focused not only on COVID-19 but also on performance support (skill acquisition) at the point of practice, which helped ensure competency in using existing technologies and tools to optimize management of the virus and comorbidities. Strategies included online courses, informal open forums, national teleconference discussions, daily meetings, and just-in-time trainings.

The level of satisfaction with these trainings, as shown in attendee evaluations (Fig. 5), was one of the indicators of their quality.

\section{STRATEGIC PARTNERSHIP: ACUTE CARE INITIATIVE}

RPM - HT initiated and led several strategic partnerships to address additional Veteran care needs during the COVID-19 pandemic. Of these partnerships, one of the most innovative and intensive was an Acute Care Initiative to enroll Veterans for COVID-19 monitoring at urgent sites of care.

Before COVID-19, most Veterans were enrolled in the RPM - HT program through primary care, specialty clinics, inpatient admissions, and mental healthcare, as well as through utilization of data reviews by RPM - HT staff who then initiated a consult for enrollment in collaboration with the Veteran's provider. Early in the COVID19 pandemic, the Office of Connected Care leadership understood that this model of enrollment would need to expand to more acute care settings, where Veterans requiring COVID19 monitoring would most likely present themselves.

The coordination of the Acute Care Initiative involved collaborations with VA's Emergency Management national program director, the COVID-19 Emergency Management Coordination Cell, the national Logistics program, the Office of Nursing Services, and other specialties.

These collaborations resulted in the development of the 'COVID-19 Remote Monitoring Strategies for Low, Moderate and High-Risk Veterans' 


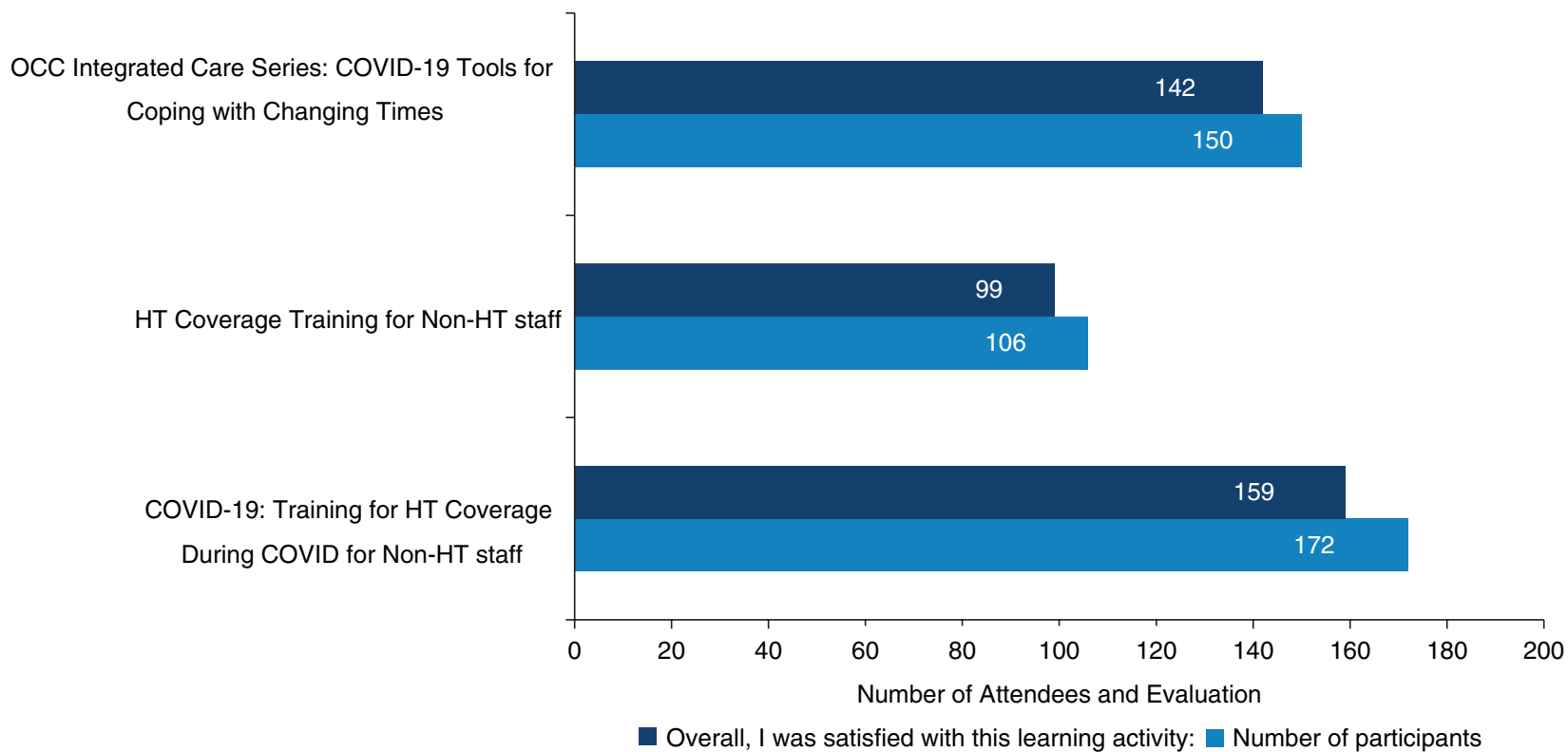

Figure 5-Attendee satisfaction with RPM-HT training, March 1-December 21, 2020.

guidance document. This document was distributed through national Emergency Management and Primary Care communications. A link to the document was also included as a reference in VA's 'Primary Care Field Guide for Managing COVID-19'.

The purpose of the guidance document was to help providers identify strategies for using and expanding remote monitoring services for Veterans with suspected or diagnosed COVID-19 illness who did not require hospitalization. Using these strategies at the point of care, such as urgent care clinics, contact centers, acute care clinics, and emergency departments, helped provide a comprehensive approach to care.

In addition, the strategies aimed to reduce admissions and preserve inpatient capacity as much as possible by remote patient monitoring. Veterans could also be given monitoring devices, such as thermometers and pulse oximeters at the point of care, to avoid any possible delay in providing the devices. This point-of-care supply was especially valuable considering delays in mail service.

The guidance document also informed staff about:

- Recommended patient classification as low risk, moderate risk, or high risk based on factors, such as age, presence of high-risk conditions, and ability to self-monitor.

- Strategies based on risk classification (Fig. 6), including low intervention, observation, case management, and case management with RPM - HT enrollment, with a specific list of steps on implementing each strategy. The strategies included use of COVID-19 selfcare monitoring programs with VA's Annie app, as well as Veteran education materials on COVID-19 monitoring.

- A streamlined checklist for emergency department or urgent care center discharge, based on the risk level, as a quick reference for staff. 


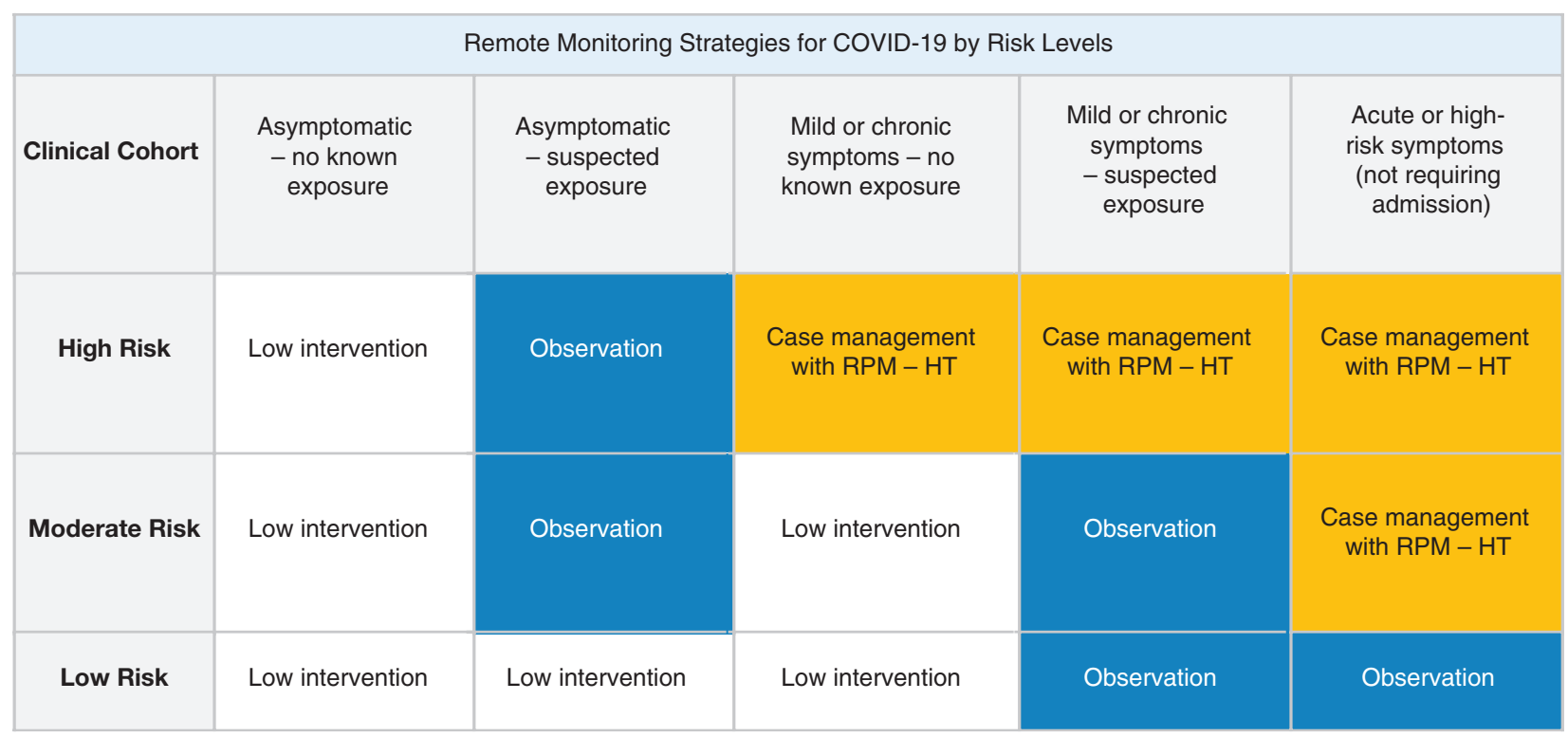

Figure 6-Table provided in the 'COVID-19 Remote Monitoring Strategies for Low, Moderate and High-Risk Veterans' guidance document. ${ }^{10}$

\section{RPM - HT OUTCOMES}

VHA's concerted efforts and actions, and its ability to build on its existing infrastructure, have enabled it to meet the daily needs for COVID-19 monitoring, including the big surges in demand, which were reported in April 2020, July 2020, and the winter of 2020-2021 (when enrollment twice exceeded 2,000 Veterans). This demand is quantified not only by the number of Veterans and other Veterans using at-home monitoring for COVID-19 but also by the need for more intensive monitoring by VHA's Care Coordinators. The average number of interventions per month for a Veteran enrolled on a COVID-19-associated DMP is substantially greater than for a Veteran enrolled on other DMPs (Fig. 7).

RPM - HT provided value to Veterans, who said they felt safe knowing that a Care Coordinator was monitoring their health. Veteran satisfaction with RPM - HT already was high before the pandemic; however, it is a testament to the success of the program's pandemic-driven efforts that patient satisfaction remained high - and in some respects inched higher - even with the need for meeting the big surge in demand in short order (Fig. 8).

Another measure of the success of the RPM - HT efforts was the ability of Veterans to continue to convalesce at home. Inpatient admissions at VA facilities from the cohort on a COVID-19-associated DMP have remained very low (Fig. 9), with the qualification that admissions to non-VA facilities were not tracked. From March 2020 to March 2021, only 4\% of Veterans on a COVID-19-associated DMP had an admission to a VA facility. Further study is needed to determine the impact RPM - HT enrollment for COVID-19 care had on the need for inpatient care.

\section{CONCLUSION}

The Office of Connected Care's successful, enterprise-wide RPM - HT business, clinical, 


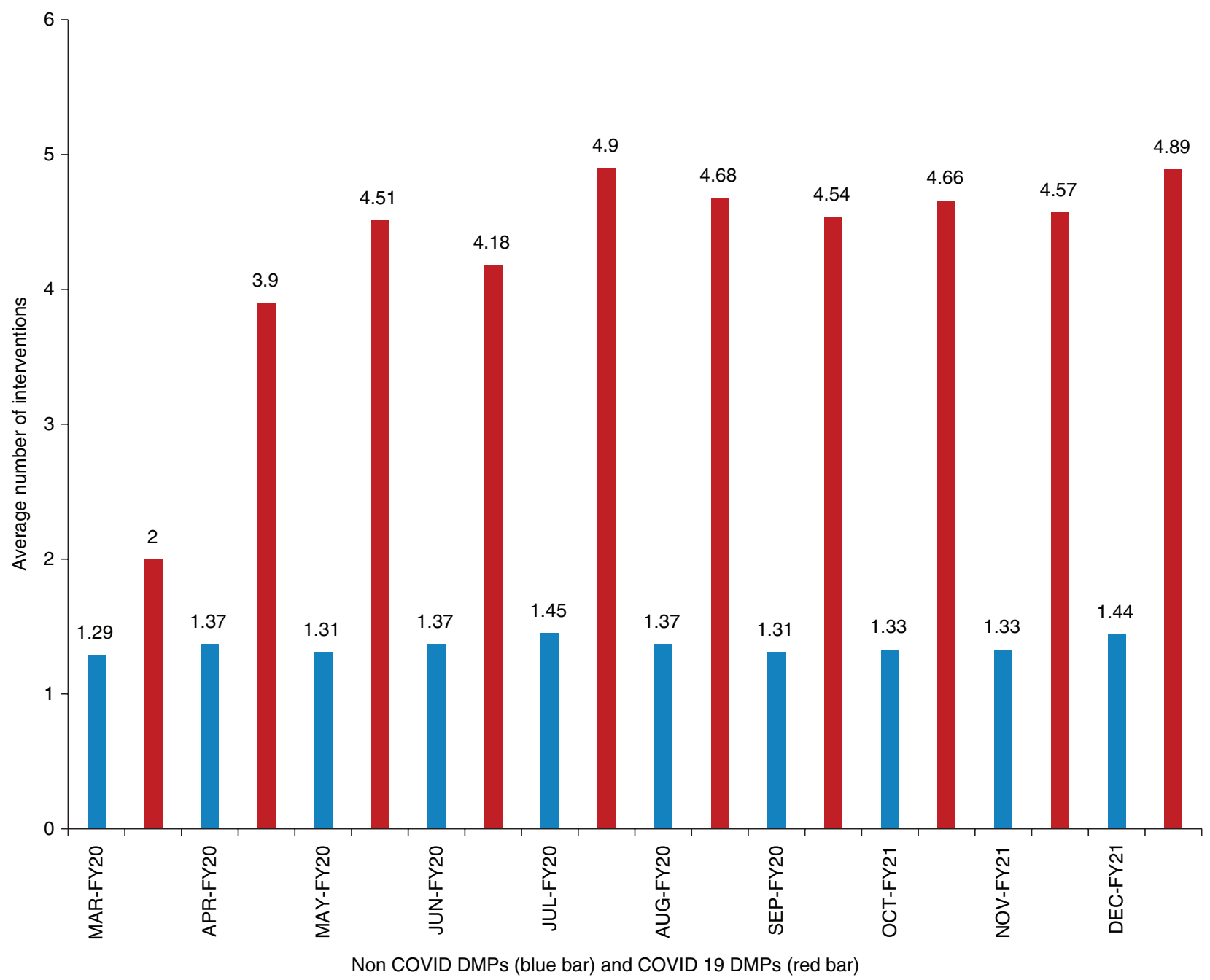

Figure 7-Average number of RPM-HT interventions per person per month for COVID-19-associated DMPs compared with all non-COVID-19 associated DMPs.

and technical infrastructure enabled VHA to enter the COVID-19 public health emergency well-positioned for the rapid deployment of and growth in RPM - HT monitoring. As the at-home management of Veterans became increasingly important during VHA's COVID-19 response, the national RPM HT program broadened its existing practices to meet the needs of Veterans, caregivers, and staff. The success of this effort demonstrates the importance of having infrastructure in place and framing it in a way that provides flexibility and allows it to be scaled up in times of greater need.

\section{CONFLICT OF INTEREST}

The study authors do not have an actual, potential, or perceived, conflict of interest in respect of the work associated with this TMT article.

\section{FUNDING}

The authors have not received any funding or benefits from the industry or elsewhere to conduct this study. 
5. During phone calls, the care given by my Care Coordinator put me at ease.

4. I feel safe knowing that my Care Coordinator is monitoring my health.

3. I know that when I submit my responses to questions and vitals, my Care Coordinator will review them.

2. If I take my vitals, submitting them each day (i.e. blood sugar, blood pressure, weight, etc.) is a simple process.

1. I trust Home Telehealth program as part of my overall VA healthcare.

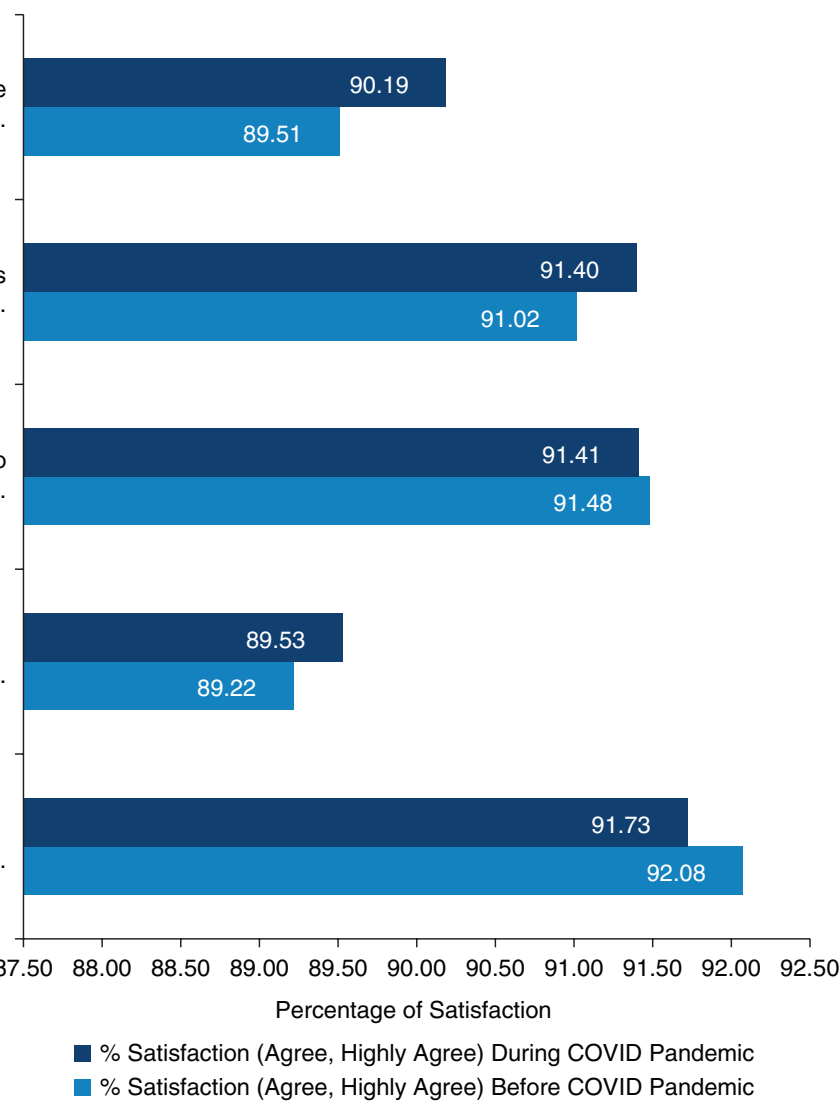

Figure 8-Veterans enrolled in RPM-HT. Pre-COVID-19 satisfaction levels measured from October 2018 to March 2020. Pandemic-period satisfaction measured from March to December 2020.

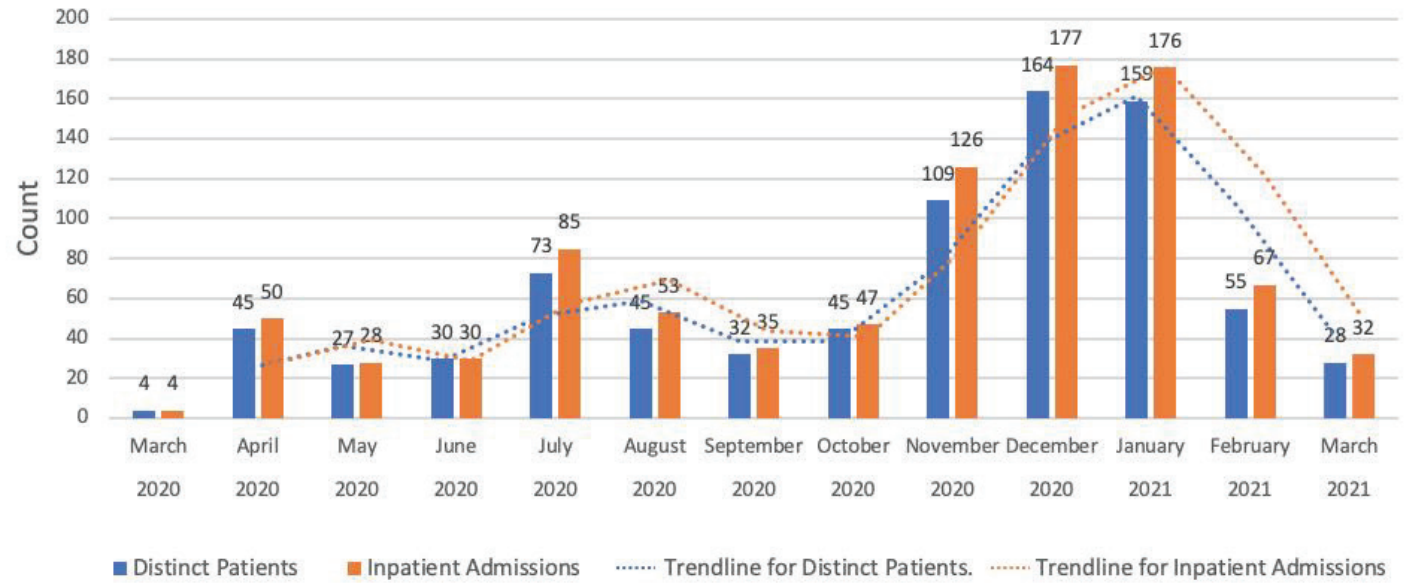

Figure 9-National inpatient admissions to VA facilities for Veterans on a COVID-19-associated DMP by number of distinct Veterans admitted and total number of admissions.

Note: The total number of inpatient admissions is higher than that of distinct Veterans because some Veterans were admitted more than once.

Telehealth and Medicine Today ${ }^{\circledR} \quad$ ISSN 2471-6960 $\quad$ https://doi.org/10.30953/tmt.v6.281




\section{References}

1. Peters J. VHA Talking points - Telehealth, Fiscal 2019. Washington, DC: U.S. Department of Veterans Affairs, Veterans Health Administration; 2019.

2. Department of Veterans Affairs COVID-19 National Summary. [Internet]. Washington, DC: U.S. Department of Veterans

Affairs; 2021. Available from: https:// www.accesstocare.va.gov/Healthcare/ COVID19NationalSummary [cited 21 May 2021].

3. Rollins A. COVID dashboard RPM-HT vendors COVID enrollment census data. Washington, DC: U.S. Department of Veterans Affairs, Office of Healthcare Transformation Division; 2021.

4. Johnston R, Kobb RF, Marty C, McVeigh P. VA video telehealth and training programs during the COVID-19 response. TMT 2021 Jan 24;6(1). doi: 10.30953/tmt.v6.241

5. Cruickshank J. Telehealth: what can the NHS learn from experience at the U.S. Veterans Health Administration. [Internet]. London: 2020health.org; 2012 Jan 6. Available from: https://www.bl.uk/collection-items/ telehealth-what-can-the-nhs-learn-fromexperience-at-the-us-veterans-healthadministration\# [cited 22 July 2021].

6. Kobb R, Chumbler NR, Rabinowitz T, Brennan D. Home telehealth: mainstreaming what we do well. Telemed J E Health 2008; 14(9): 977-81. doi: 10.1089/tmj.2008.0121

7. Darkins A, Ryan P, Kobb R, Foster L, Edmonson E, Wakefield B, et al. Care coordination/home telehealth: the systematic implementation of health informatics, home telehealth and disease management to support the care of veteran patients with chronic conditions. Telemed J E Health 2008; 14(9): 1118-26. doi: 10.1089/tmj.2008.0021

8. Meyer M, Ryan P, Kobb R, Roswell R. Identifying veterans' needs for home telehealth. Fed Pract 2003; 20(8): 24-41.

9. Meyer M, Kobb R, Ryan P, Gokalp H. Virtually healthy: chronic disease management in the home. Dis Manag 2004 Jul 5; 5(2): 87-94. doi: $10.1089 / 109350702320229186$

10. Use of remote patient monitoring Home Telehealth technologies for COVID-19. [Internet]. Washington, DC: U.S. Department of Veterans Affairs; 2020 Mar 25. Available from: https:// dvagov.sharepoint.com/:w:/r/sites/ VACOVHAPublicHealth/HCI/_layouts/15/ Doc.aspx? sourcedoc $=\% 7 \mathrm{~B} 44773601$ EBC2-4826-975BD106BB4DD3E2\%7D\&file $=$ Primary $\% 20$ Care\%20Field\%20Guide\%20 for $\% 20$ Managing $\% 20$ COVID19 FINAL $\% 20$ to $\% 2010 \mathrm{~N}$. docx\&action $=$ default\&mobileredirect $=$ true.

Copyright Ownership: This is an open access article distributed in accordance with the Creative Commons Attribution Non Commercial (CC BY-NC 4.0) license, which permits others to distribute, adapt, enhance this work non-commercially, and license their derivative works on different terms, provided the original work is properly cited and the use is noncommercial. See: http://creativecommons. org/licenses/by-nc/4.0. 